\title{
Glycolipid Ranking of Bread Quality Hard Wheat Breeding Stock Cultivars by Tandem Mass Spectrometry of Total Lipid Extract
}

\author{
M.D. Boatwright ${ }^{1,2}$, A.K. FritZ ${ }^{3}$ and D.L. WeTZEL ${ }^{1 *}$ \\ ${ }^{1}$ Microbeam Molecular Spectroscopy Laboratory, \\ ${ }^{2}$ Department of Biochemistry and Molecular Biophysics, \\ ${ }^{3}$ Department of Agronomy, Kansas State University, Manhattan, KS, 66506 USA \\ (Received 12 November 2016; Accepted 15 December 2016; \\ Communicated by F. Békés)
}

\begin{abstract}
Desirable wheat glycolipid routine analysis for the first time enables selection for a significant quality factor among bread quality hard wheat experimental cultivars. Prior to producing sufficient quantity of later generation experimental wheats to allow milling and baking, objective chemical testing of the protein and lipid components of the endosperm can make the wheat breeding process more efficient by earlier elimination of cultivars with inferior quality traits. Tandem mass spectrometric (MS-MS) analysis of digalactosyldiglycerides (DGDG) from total wheat endosperm lipid extract is now suitable as a routine desirable lipid ranking tool. This automated multistep technique eliminates time consuming fractionation, selective extraction, and chromatographic procedures of the past decades that resulted in limited capacity precluding extensive routine application.
\end{abstract}

Keywords: tandem mass spectrometry, glycolipids, digalactosyldiglycerides, wheat breeding, breadmaking quality wheat

\section{Introduction}

The need for nonsubjective analytical chemical data relative to the glycolipid content of early generations of bread quality hard wheat cultivars has existed for at least three decades since pioneering wheat lipid work was carried out by experts in experimental baking. Early investigations established the lipid contribution as nature's surface active agent to envelope the fermentation product thereby enhancing the loaf volume. Subsequently, investigators developed intricate extraction, fractionation, and chromatographic techniques to separate various glycolipids from nonpolar glycerides and phospholipids found in the wheat endosperm.

Thin layer chromatography (TLC) prior to 1981 was the primary analytical technique for wheat lipid studies in regard to their influence on breadmaking. Among the wheat lipid classes: nonpolar, glycolipid, and phospholipid, four diglycerides from the glycolipid class were separated. Prior to TLC separation, the nonpolar fraction was selectively ex- 
tracted and the phospholipid fraction was retained on a silica column after elution of the glycolipid as the petroleum ether polar extract. Glycolipid lipid research of the TLC era was cited extensively (Pomeranz 1971; Chung and Tsen 1975; Chung et al. 1978; MacRitchie 1977; Christie and Morrison 1988). In particular, OK Chung related baking loaf volume to the indicative digalactosyldiglyceride (DGDG) content of various wheat cultivars. The wheat polar lipid (or specifically glycolipid) contribution to breadmaking has been the subject of numerous papers prior to or concurrent with the separation of individual contributing glycolipids (Bell et al. 1979, 1986; Zawistowska et al. 1986).

With the application of high performance liquid chromatographic (HPLC) separation and subsequent mass spectrometric analysis of chromatographic fractions by Tweeten et al., various individual glycolipid compounds were revealed and quantitated (Tweeten 1979; Tweeten et al. 1981). This achievement was recognized via an honored student award by the American Oil Chemists' Society at their San Francisco 1979 AOCS annual meeting. For the flour milled from 23 identity preserved well characterized hard wheat specimens, HPLC determination of digalactosyldiglyceride (DGDG) percent of total lipid varied between 11.6 and 3.0 (Tweeten 1979). Also, the monogalactosyldiglceride (MGDG) varied between 6.5 and 1.8 percent. Regrettably, a 22 hour procedure was required by the combined extraction, fractionation, and HPLC analysis. Subsequently, a correlation between the petroleum ether polar extraction fraction and baking loaf volume was reported (Chung et al. 1982) that supported the significance of DGDG determination. An effort to accelerate the HPLC was introduced (Ohm and Chung 1999), however, the changing solvent baseline precluded direct linear spectroscopic detector usage. Numerous research groups studied the contribution of polar lipid or free lipid to breadmaking at this time (Larsen et al. 1989; Kárpáti et al. 1990; McCormack et al. 1991a, 1991b; Panozzo et al. 1990, 1993), which has since been thoroughly summarized (Chung et al. 2009).

Nearly a decade passed before an accelerated supercritical fluid, two step extraction procedure was developed at KSU that isolated the polar fraction of each successive specimen every 15 minutes to allow subsequent automated HPLC or supercritical fluid chromatographic (SFC) separation and determination of the various glycolipids of interest. This was achieved by PhD student Ming Zhao to at long last enable a practical, routine analytical procedure (Zhao 1997). A dedicated tandem oven, tandem 6000 psi pump systems apparatus was constructed that with high pressure valving enabled 15 minute sequential pure supercritical fluid extraction (SFE) with $\mathrm{CO}_{2}$ followed by a $4 \%$ methanol/ $\mathrm{CO}_{2}$ supercritical fluid cocktail to enable removal of the nonpolar lipid fraction and isolation of polar lipid fractions (Wetzel et al. 2005). Local financial constraints resulted in termination of routine annual glycolipid determination in support of the wheat breeding program. Regrettably, the thorough analytical methods that involved a succession of steps with manual involvement at various stages were too time consuming to enable screening of experimental cultivar nurseries, and thus had only a limited impact on the Kansas wheat breeding efforts.

After a period of not being able to perform glycolipid analysis on new wheat cultivars by chromatographic procedures, the rise in capability of the KSU lipidomics laboratory 
in the biology department under Dr. Ruth Welti made advanced analytical techniques possible. Once the labor intensive sample preparation was done and each batch of total lipid extracts was frozen, it was possible to have automated quadrupole analysis that did not require selective extraction, fractionation, or HPLC. As a result, the chemical composition of individual glycolipids is readily determined. This enables the causality of favorable lipid contribution to be determined relative to one aspect of baking quality, whereas in the past the result of the glycolipid contribution was reported as loaf volume. Presently, total lipid extracts from wheat endosperm are selectively analyzed by tandem mass spectrometry (MS-MS) with a triple quadrupole mass spectrometer. Selection of precursor molecule ions by RF programming, collision chamber loss of galactose units, and selective reporting of the acyl molecule ions of interest from an automated MS-MS operation yield sufficient data to enable ranking of experimental cultivars of Kansas wheat breeding generations for glycolipids on a practical basis.

\section{Materials and Methods}

Mass spectrometric analysis of molecule ions produced by the soft ionization technique of electrospray was used to analyze the lipid composition of wheat lipid total extracts. An Applied Biosystems 4000 Q-TRAP tandem mass spectrometer (Grand Island, NY) was used to analyze total lipid extracts and acquire data specifically for glycolipids with precursor ion scanning. After electrospray ionization, the first quadrupole of the mass spectrometer was employed to scan the specified mass/charge $(\mathrm{m} / \mathrm{z})$ range. An auxiliary quadrupole was a collision chamber containing nitrogen gas to induce fragmentation. The third quadrupole subsequently scanned radio frequencies at an $\mathrm{m} / \mathrm{z}$ offset of one (179 $\mathrm{m} / \mathrm{z})$ or two $(341 \mathrm{~m} / \mathrm{z})$ galactose units. We previously used the same procedure on small specimens from hand dissected wheat kernels (Brewer 2009; Wetzel et al. 2014; Brewer and Wetzel 2016). The samples prepared each day were immediately frozen in the autoloader vials and subsequently submitted for MS-MS. All results cited were obtained on the specimen extracts along with a set of quantitative standards.

A total of 433 flour samples from individual cultivars of various F7 and F8 nursery experimental wheats were subjected to total lipid extraction and prepared for MS-MS glycolipid analysis. An adaptation of the method previously reported by Welti et al. (2002, 2003) was used for sample preparation and the MS-MS operation. For details of the total lipid extraction procedure and the stepwise preparation of individual specimens for eventual automated MS-MS analysis for DGDG as a multiple sample batch, refer to the following website (http://www.k-state.edu/lipid/lipidomics/service.htm).

\section{Results}

The histogram of Fig. 1 shows the DGDG distribution among a single MS-MS autoloader overnight batch run. The individual autoloader samples were frozen immediately after the transfer of extract to the autoloader vial and addition of an internal standard. The accumulated frozen specimens pertaining to Figure 1 were submitted in this case as a single 


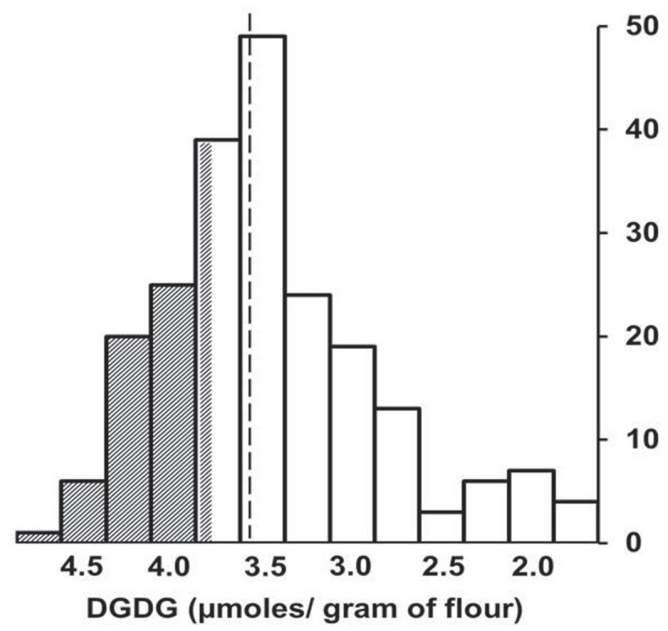

Figure 1. Distribution of DGDG content from analyses of an automated MS-MS batch run. The median of 4.36 $\mu$ moles DGDG/gram of flour is indicated by the dashed line. The shaded area indicates the highest $35 \%$, which if selected for retention will have a minimum of $3.85 \mu$ moles DGDG/g of flour. The open area indicates a trailing extended range

batch of 215. A total of 433 experimental hard wheat endosperm cultivars were analyzed. The top 35\% of each MS-MS batch run was retained for a total of 143 out of 433 analyzed in three separate batches. A previous experiment (Wetzel et al. 2014) used later generations, available in sufficient quantity, to study the statistical replication of MS-MS quantitation. Seven replicates for four distinct levels of DGDG containing specimens were subjected to the preliminary extraction and MS-MS. For specimens with concentrations of $3.35,2.94,2.52$, and $2.40 \mathrm{nmol} / \mathrm{mg}$ of flour for the DGDG lipid component, the respective relative standard deviations were $3.9,5.4,5.6$, and $4.6 \%$.

\section{Discussion}

Elimination of the manual lipid fractionation step prior to mass spectrometric glycolipid determination has increased the capacity to screen experimental nursery products. The glycolipid determination of desirable glycolipid content in 433 experimental hard wheat endosperm cultivars enabled identification of the top $35 \%$ to be retained for a total of 143 . It should be noted that prior to selection of cultivars for glycolipid analysis, subjective examination eliminated seeds that did not exhibit primary agronomic quality criteria. Selection of experimental breeding lines to be carried forward from early generations has traditionally been highly dependent on expert subjective judgment by the wheat breeder in regard to multiple criteria. Due to the small specimen requirement of this analytical procedure related to quality for end use, a numerical objective criterion of quality is now available for routine application. 
Previous research involving wheat lipids has been reported for various purposes. Geng et al. (2015) and Gonzalez-Thuiller et al. (2015) used MS applied to wheat glycolipids; however, they followed the usual route of doing so only after prior laboratory fractionation procedures. Guella et al. (2003) suggested the use of ESI-MSMS for wheat glycolipid determination, but did not actively apply the technique to wheat endosperm. Recently, Moore et al. (2015) employed NMR for quantitation of total lipid in wheat. They used the results to successfully identify genetic markers for the caloric contribution of lipid content to the diet whereas the objective of our work has been concerned with the effect of the select glycolipid fraction on loaf volume for experimental wheat cultivars.

Selection within wheat breeding programs for quality still focuses largely on phenotypic screening and assessment of high molecular weight glutenins. Quality is clearly a complex trait and designing end use quality for specific products or uses will require a more detailed knowledge of all components of quality. The current work clearly demonstrates that genetic variation exists for the lipid profile within an active breeding program. Previous work has shown these compounds do have an impact on loaf volume (Chung et al. 1982). The ability to accurately profile lipids allows for inclusion of these compounds in wheat breeding selection criteria. Many programs are working toward using genomic selection for prediction of end use quality (Battenfield et al. 2016). The use of phenotypic data, such as loaf volume, can be problematic as multiple factors influence the trait. The ability to use more primary traits, such as lipid composition, in the selection models should make the models much more robust.

\section{Acknowledgements}

The extensive efforts of previous wheat workers and the subsequent adaptation of developing analytical techniques by individuals whose names appear in the references are hereby recognized. The authors thank Tyler Nickoley for technical assistance with MSMS and Dr. Rebecca Miller who furnished the experimentally milled identity preserved flour specimens. Contribution no. 17-108-J of the Kansas Agricultural Experiment Station, Manhattan.

\section{References}

Battenfield, S.D., Guzman, C., Gaynor, R.C., Singh, R.P., Pena, R.J., Dreisigacker, S., Fritz, A.K., Poland, J.A. 2016. Genomic selection for processing and end-use quality traits in the CIMMYT spring bread wheat breeding program. Plant Genome 9:1-12.

Bell, B.M., Chamberlain, N., Collins, T.H., Daniels, D.G.H., Fisher, N. 1979. The composition, rheological properties and breadmaking behavior of stored flours. J. Sci. Food Agric. 30:1111-1122.

Bekes, F., Zawistowska, U., Zillman, R.R., and Bushuk, W. 1986. Relationship between lipid content and composition and loaf volume of twenty-six common wheats. Cereal Chem. 63:327-331.

Brewer, L.R. 2009. IR microspectroscopic imaging discriminates isogenic null waxy from parent wheats with lipid class profile supported by compositional analyses. MS Thesis. Kansas State University. Manhattan, KS, USA.

Brewer, L.R., Wetzel, D.L. 2016. Tandem MS characterization of endosperm lipid profile in isogenic waxy wheat versus wildtype parent cultivars. J. Am. Oil Chem. Soc. 93:751-756. 
Christie, W.W., Morrison, W.R. 1988. Separation of complex lipids of cereals by high-performance liquid chromatography with mass detection. J. Chromatogr. 436:510-513.

Chung, O.K., Tsen, C.C. 1975. Changes in lipid binding and distribution during dough mixing. Cereal Chem 52:533.

Chung, O.K., Pomeranz, Y., Finney, K.F. 1978. Wheat flour lipids in breadmaking. Cereal Chem. 55:598.

Chung, O.K., Pomeranz, Y., Finney, K.F. 1982. Relation of polar lipid content to mixing requirement and loaf volume potential of hard red winter wheat flour. Cereal Chem. 59:14-20.

Chung, O.K., Ohm, J.-B., Ram, M.S., Park, S.-H., Howitt, C. 2009. Wheat lipids. In: Khan, K., Shewry, P.R. (eds), Wheat Chemistry and Technology. 4th Edition. AACC Press. St Paul, MN, USA. pp. 363-400.

Geng, P., Harnly, J.M., Chen, P. 2015. Differentiation of whole grain from refined wheat (T. aestivum) flour using lipid profile of wheat bran, germ, and endosperm with UHPLC-HRAM mass spectrometry. J. Agric. Food Chem. 63:6189-6211.

González-Thuillier, I.G., Salt, L., Chope, G., Penson, S., Skeggs, P., Tosi, P., Powers, S.J., Ward, J., Wilde, P., Shewry, P.R., Haslam, R.P. 2015. Distribution of lipids in the grain of wheat (cv. Hereward) determined by lipidomic analysis of milling and pearling fractions. J. Agric. Food Chem. 63:10705-10716.

Guella, G., Frassantio, R., Mancini, I. 2003. A new solution for an old problem: the regiochemical distribution of the acyl chains in galactolipids can be established by electrospray ionization tandem mass spectrometry Rapid Commun. Mass Spectrom. 17:1982-1994.

Kárpáti, M., Békés, F., Smied, I., Lásztity, R., Mosonyi, A., Örsi, F. 1990. Investigation of the relationships between wheat lipids and baking properties. Acta Alim. 19:237-260.

Larsen, N.G., Humphrey-Taylor, V.J., Baruch, D.W. 1989. Glycolipid content as a breadmaking quality determinant in flours from New Zealand wheat blends. J. Cereal Sci. 9:149-157.

MacRitchie, F. 1977. Flour lipids and their effects in baking. J. Sci. Food Agr. 28:53.

McCormack, G., Panozzo, J., MacRitchie, F. 1991. Contributions to breadmaking of inherent variations in lipid content and composition of wheat cultivars. I. Results of survey. J. Cereal Sci. 13:225-261.

McCormack, G., Panozza, J., MacRitchie, F. 1991. Contributions to breadmaking of inherent variations in lipid content and composition of wheat cultivars. II. Fractionation and reconstitution studies. J. Cereal Sci. 13:263-274.

Moore, C.M., Richards, R.A., Rebetzke, G.J. 2015. Phenotypic variation and QTL analysis for oil content and protein concentration in bread wheat (Triticum aestivum L.). Euphytica 204: 371-382.

Ohm, J.B., Chung, O.K. 1999. Estimation of free glycolipids in wheat flour by HPLC 1. Cereal Chem 76:873876.

Panozzo, J.F., O'Brien, L.O., MacRitchie, F., Bekes, F. 1990. Baking quality of Australian wheat cultivars varying in their free lipid composition. J. Cereal Sci. 11:51-57.

Panozzo, J.F., Hannah, M.C., O'Brien, L, Bekes, F. 1993. The relationship of free lipids and flour protein to breadmaking quality. J. Cereal Sci.17:47-62.

Pomeranz, Y. 1971. Composition and functionality of wheat flour components. In: Pomeranz, Y. (ed.), Wheat: Chemistry and Technology. Am. Assoc. of Cereal Chem., St. Paul, Minnesota, USA. p. 585.

Tweeten, T.N. 1979. Analytical high performance liquid chromatography of digalactosyl diglyceride in wheat flour lipid fractions. PhD Dissertation. Kansas State University. Manhattan, KS, USA.

Tweeten, T.N., Wetzel, D.L., Chung, O.K. 1981. Physiochemical characterization of galactoysyldiglycerides and their quantitation in wheat flour lipids by high performance liquid chromatography. J. Am. Oil Chem. Soc. 58:664-672.

Welti, R., Li, W., Li, M., Sang, Y., Biesiada, H., Zhou, H., Rajashekar, C.B., Williams, T.D., Wang, X. 2002. Profiling membrane lipids in plant stress responses: Role of phospholipase $\mathrm{D}\{\mathrm{alpha}\}$ in freezing-induced lipid changes in arabidopsis. J. Biol. Chem. Soc. 277:31994-32002.

Welti, R., Wang, X., Williams, T.D. 2003. Electrospray ionization tandem mass spectrometry scan modes for plant chloroplast lipids. Anal. Biochem. 314:149-152.

Wetzel, D.L., Zhao, M., Sweat, J.A., Wilson, R. 2005. Tandem two-step supercritical fluid, selective extraction system for polar lipid recovery from wheat flour. In: Chung, O.K., Lookhart, G.L. (eds), Proc. $3^{\text {rd }}$ Int. Wheat Quality Conf. Grain Industry Alliance. Manhattan, KS, USA. pp. 183-201. 
Wetzel, D.L., Boatwright, M.D., Fritz, A.K. 2014. Tandem mass spectrometric determination of glycolipids in wheat endosperm: a new tool for breeders to rank and select early seed generations. J. Am. Oil. Chem. Soc. 91:1849-1855.

Zawistowska, U., Bekes, F., Bushuk, W. 1986. Intercultivar variations in lipid content, composition, and distribution and their relation to baking quality. Cereal Chem. 61:527-531.

Zhao, M. 1997. Selective supercritical fluid extraction of wheat flour glycolipids and their determination by supercritical fluid chromatography, Ph.D. Dissertation, Kansas State University. Manhattan, KS, USA. 